\title{
Laparoscopic management of Dermoid Cyst with Elevated Carbohydrate antigen 19-9
}

\author{
Siraj SHM , Sui Min Bernard
}

\section{KEY WORDS}

Carbohydrate antigen CA 19-9; Tumor markers; Dermoid cyst; Mature cystic teratoma.

\section{INTRODUCTION}

Serum carbohydrate antigen CA 19-9 (also called as cancer antigen 19-9; sialylated Lewis (a) antigen) was initially described in gastrointestinal adenocarcinomas. However, it can be detected in other malignancies and in some benign conditions as well. Dermoid cyst, also called as mature cystic teratoma (MCT) or benign cystic teratoma of the ovary, is the most common germ cell tumor of the ovary in reproductive aged women. ${ }^{1}$ It accounts for $10-25 \%$ of all ovarian neoplasms and $60 \%$ of all benign ovarian tumors. ${ }^{2}$ Malignant transformation occurs in only 1-3\% of cases, especially in postmenopausal women. $15 \%$ of cases develop torsion; however, rupture is rare probably due to thick cyst wall. It is mostly common in women aged between 20 and 40 years. ${ }^{3}$ Increased CA 19-9 levels in some Dermoid ovarian cysts have been reported. ${ }^{4}$ The present study is reporting a laparoscopic management of a Dermoid cyst with elevated CA 19-9.

\section{CASE}

A 29 -year old lady presented to our clinic with incidental finding

Correspondence:

E-mail:

Competing interests: None of raised CA 19-9 (216U/ML) which was detected through her routine cancer screening. She was asymptomatic and Abdominal pelvic ultrasonography revealed $8.7 \times 8.6 \mathrm{~cm}$ heterogeneous cystic left adnexal mass with bright echogenic areas compatible with Dermoids (Figure 1 ). The right ovary and other intraabdominal structures were normal. Other tumor markers were within normal range (Carcinoembryonic antigen (1.8 ug/L), CA 125 (16.5U/ Ml), Beta-Hcg<1.2, Alphafoeto protein $(4 \mathrm{ug} / \mathrm{L})$. Other possible gastrointestinal system pathology was ruled out by imaging and endoscopic procedures. Laparoscopic cystectomy was performed (Figure 2, 3) and a histopathological evaluation of a frozen section that Cyst was the dermoid cyst. Multiple sections from various cyst sites did not reveal any malignant foci. The cyst wall was lined by squamous epithelium. There were also pieces of tissue lined by squamous epithelium and containing sebaceous and sweat glands, adipose tissue, smooth muscle, glial tissue, choroid plexus, nerve bundles, cysts lined by respiratory type epithelium, cartilage and melanocytes.no immature elements nor malignancy seen and it also a cyst with fibrous wall which shows numerous spaces partly surrounded by macrophages resembling a lipogranulomatous reaction to extravasated cyst content. it was compatible with ulcerated benign cystic mature teratoma. Post operatively tumor markers returned to normal level.(11.9U/ML)

\section{DISCUSSION}

Teratomas may occur at any age but the peak incidence is reported between 20 and 40 years of age. ${ }^{5}$ The adnexal mass identified in children and adolescents showed different clinical manifestations, such as abdominal pain and distention. ${ }^{6,7}$ Although the Dermoid Cysts are frequently asymptomatic and

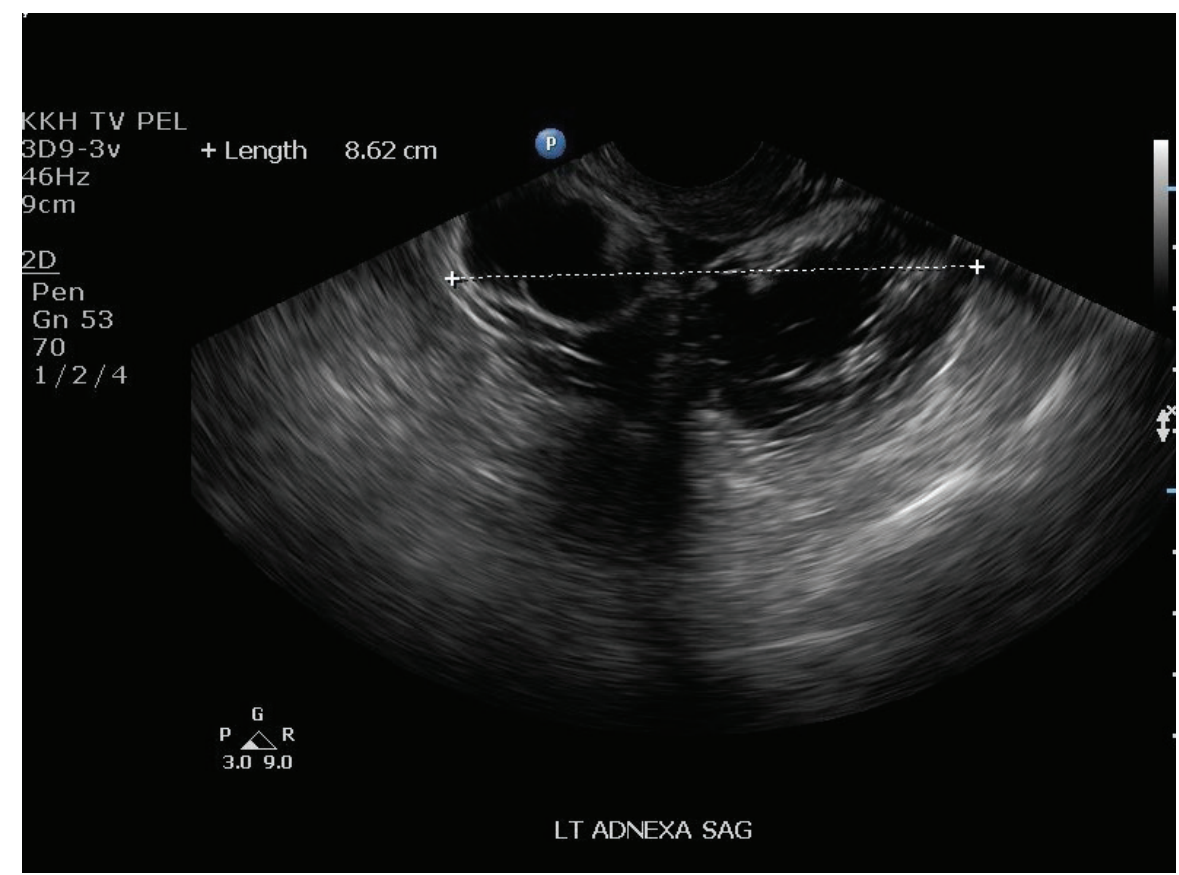

Figure 1: Heterogeneous cystic left adnexal mass with bright echogenic areas compatible with Dermoids 


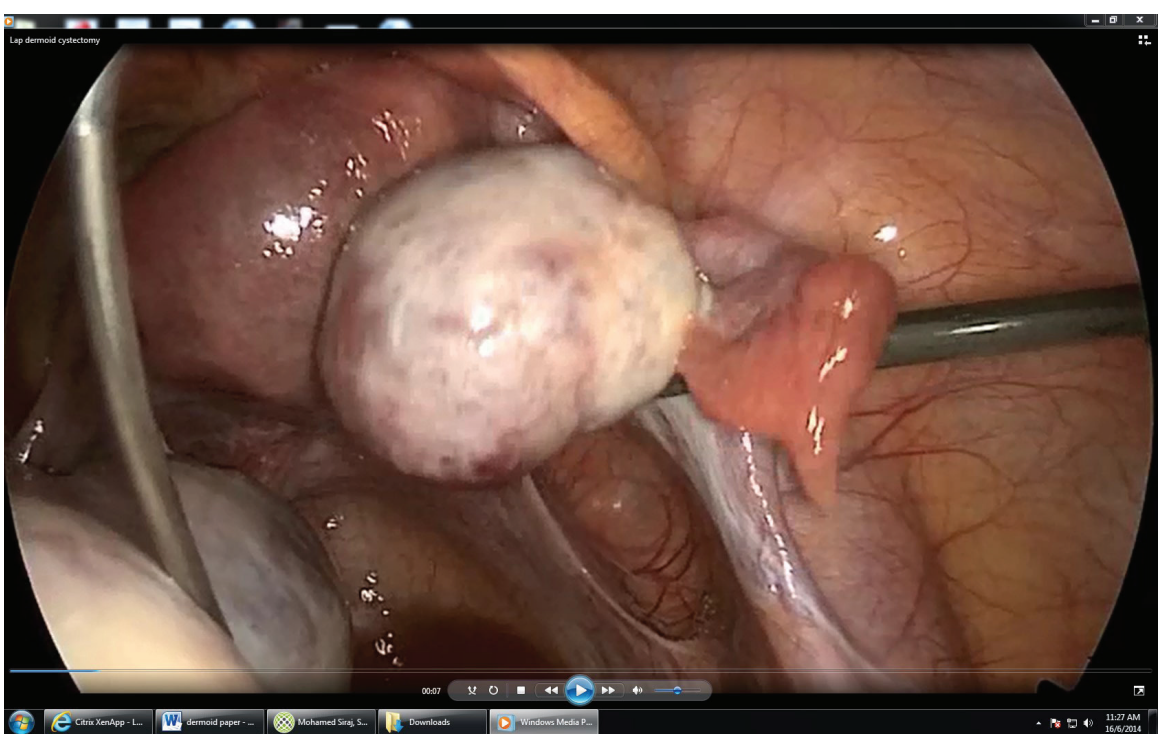

Figure 2: Laparoscopic appearance of the normal ovary

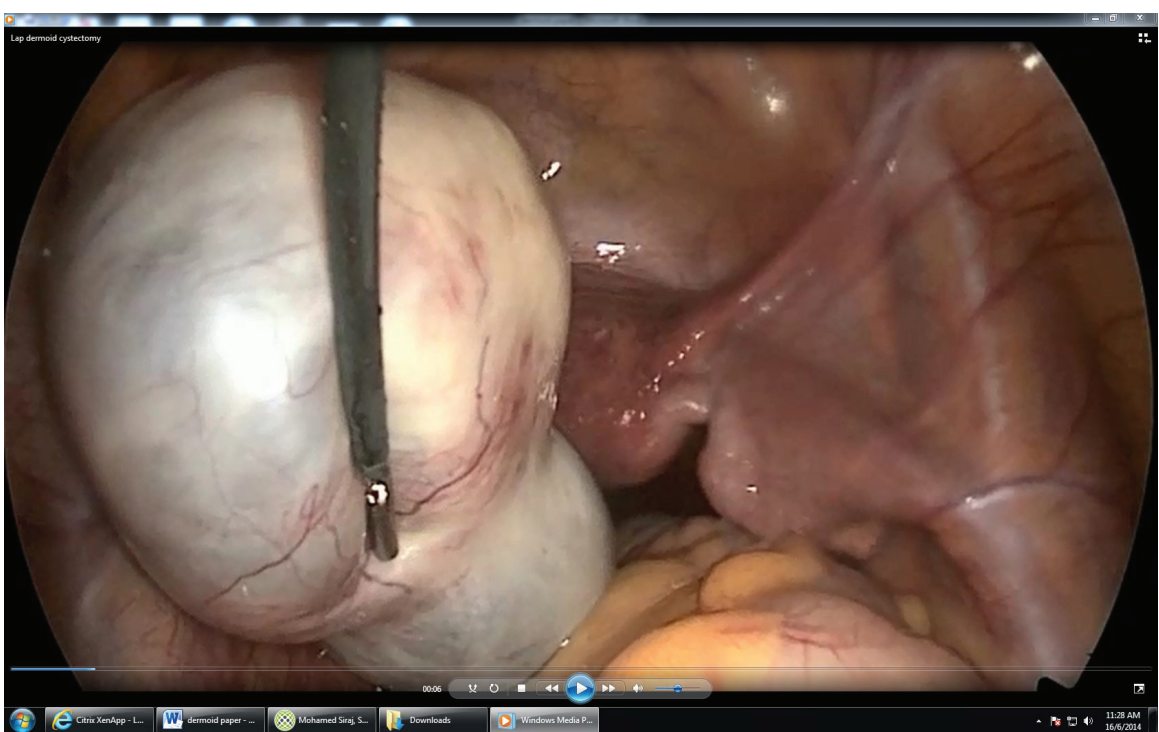

Figure 3: Laparoscopic appearance of the Dermoid cyst

detected incidentally, approximately $20 \%$ of cases were reported clinical attention with complications. Ovarian teratomas are often discovered as an incidental finding on physical examination, imaging procedures, or during abdominal or pelvic surgery performed for other reasons.

Because the surgical procedure performed is quite different for benign and malignant tumors, differential diagnosis is very important for women with an ovarian mass. Despite the specific features of MCT, including fat component and calcification, making a differential diagnosis is sometimes very difficult even with pelvic imaging studies. According to a report by Mais et al. nearly 30\% of MCT may not be apparent in sonography, due to the presence of associated pelvic abnormalities (e.g., endometriomas, large fibromas, or large contralateral ovarian masses) or intrinsic sonographic characteristics of MCTs. ${ }^{8}$ Actually, $80 \%$ of cystic teratomas were reported to have an echo pattern that should suggest malignancy. ${ }^{9}$ In addition, other features of MCT, such as a very large tumor size and significantly elevated tumor markers could make the diagnosis more difficult.

Few tumor markers such as CA 19-9 and CA 125 have been the topics of recent studies pointing that CA 19-9 may be related to the existence of the Dermoid cyst. ${ }^{10,11}$ CA125 is known to be expressed in Coelomic epithelium, including Müllerian epithelium, peritoneum, pleura, and pericardium. ${ }^{12}$ Considering the wide distribution of CA 125 expression, elevations of serum CA125 in various benign and inflammatory conditions, including MCT, are not unexpected. Even though the combination of CA125 level and imaging studies raised the early detection rates of ovarian malignancy, sometimes more information is required to distinguish MCT from Ovarian carcinoma. ${ }^{13,14}$ Difficulty in the differential diagnosis of MCT from Ovarian cacinoma usually stems from the morphologic and serologic characteristics of MCT, including large size and elevations of various tumor markers.

It was reported that Immuno histochemically, CA19-9 staining was prominent on the apical cyto-plasm of the epithelial lining, not on the basement membrane side, suggesting that CA 19-9 was secreted into the cystic cavity and concentrated there. Unless the cyst is ruptured, such a high level of CA 19-9 is not expected in the general circulation ${ }^{1}$ Yet, benign inflammatory diseases of the gastrointestinal system are known to cause CA 19- 9 elevations, and any inflammation of Dermoid cyst tissues could be a possible cause of CA 19-9 leakage from cystic cavities into the bloodstream (Rupture,Torsion and Ulceration of Dermoid cyst)

Approximately 1-3\% of dermoid cysts can be complicated with malignant transformation. However, CA 125 and CA 19-9 levels appear inconsequential with squamous cell carcinoma arising from Dermoid cyst. ${ }^{15}$ In a study consisting of 215 cases, CA 19-9 levels were elevated in $40 \%$ of selected population with mature cystic teratoma. Mean serum levels of CA 19-9 were $83.5 \pm 179.2 \mathrm{U} / \mathrm{mL}^{12}$ CA 125 levels with the mean 26.2 $\pm 29.9 \mathrm{U} / \mathrm{mL}$ were significantly higher in patients with elevated CA 19-9. ${ }^{12}$ In an study including 250 patients, 31 cases had CA 19-9 above 101U/ mL. ${ }^{13}$ Serum levels of CA 19-9 were 
correlated with the tumor size [8-11]. Bilaterality was debated regarding the extent of elevation. ${ }^{8,9}$ Correlation between Bilaterality and elevation of CA 19-9 was found only in one study. ${ }^{9}$ Concomitant elevation of CA 125 and CA 19-9 was only found in 3\% $(5 / 163)$ cases. ${ }^{10}$ However, the clinical significance with elevated CA 19-9 levels is still controversial.

A recent study by Kyung et al. postulated that CA 19-9 may be a predictor of the ovarian torsion. The mean serum CA 19-9 levels of 21 cases with ovarian torsion were $37.2 \mathrm{U} /$ $\mathrm{mL}$ (range: 14.1-570.0), whereas mean serum CA 19-9 levels of 21 cases without ovarian torsion were $20.2 \mathrm{U} / \mathrm{mL}(\mathrm{p}=0.0062) \cdot{ }^{15}$ Elevated CA 19-9 levels May also be a result of the rupture of the dermoid cyst leading to leakage from cyst wall into the bloodstream. ${ }^{2}$ A weakened cyst wall due to large diameter of the cyst may be another cause of the elevated CA 19-9 level. ${ }^{2}$ The elevated CA 125 level in dermoid cyst may be due to peritoneal involvement such as pelvic inflammatory disease or endometriosis. ${ }^{1}$ With the torsion of the ovary, an inflammatory process may be the cause of great elevation of CA-125. The cyst wall may become weaker and weaker by the torsion leading to greater increase in serum levels of CA 19-9.

The Gynecologists are disturbed by the High levels of tumor markers in the differential diagnosis between benign and malignant ovarian tumors. Serum elevations of these tumor markers do not necessarily point to a malignancy even when they reach extremely high levels. it may be due to rupture, torsion or even may be due to ulceration of Dermoid cyst as in our case. Adnexal masses with elevated CA 19-9 could be managed laparoscopically However, a complete preoperative and intraoperative workup to detect any sign of malignancy is necessary.

\section{REFERENCES}

1. Yoshioka $T$ and Tanaka $T$ "immunohistochemical and molecular studies on malignant transformation in mature cystic teratoma of the ovary",Journal of Obstetrics and Gynaecology Research, vol. 24, no. 2, pp.83-99,1998.

2. Atabekoglu C, Bozaci EA, and Tezcan S, "Elevated carbohydrate antigen 19-9 in a dermoid cyst," International Journal of Gynecology and Obstetrics, Vol. 91, no. 3, pp. 262-263, 2005.

3. Emin U, Tayfun G, Cantekin I, Ozlem UB, Umit B, and Leyla M, "Tumor marker in mature cystic teratomas of the ovary", Archives of Gynecology and Obstetrics, vol.279, no. 2, pp.145-147, 2009.

4. Uyama T, Monden Y, Sumitomo M, Miura K, Kimura S. CEA and CA 19-9 in benign pulmonary or mediastinal cystic lesions. J Surg Oncol 1989;41:103-8.

5. Lakkis WG, Martin MC, Gelfand MM. Benign cystic teratoma of the ovary: a 6 year review. Can J Surg 1985;28:444-6.

6. Kozlowski K. Management quandary. Adnexal masses in teenagers. J Pediatr Adolesc Gynecol 2000;13:145-6.

7. Pfeifer SM, Gosman GG. Evaluation of adnexal masses in adolescents. Pediatr Clin North Am 1999;46:573-92.

8. Mais V, Guerriero S, Ajossa $S$ et al: Transvaginal ultrasonography in the di-agnosis of cystic teratoma. Obstet Gynecol, 1995; 85: 48-52

9. Quinn Sf, Erickson S, Black WC: Cystic ovarian teratomas: the sonographic appearance of the dermoid plug. Radiology, 1985; 155: 477-78

10. U. Emin, G. Tayfun, I. Cantekin, U. B. Ozlem, B. Umit, and M. Leyla, "Tumor markers in mature cystic teratomas of the ovary," Archives ofGynecology andObstetrics, vol. 279, no.2,pp. 145147, 2009

11. M. Dede, S.Gungor,M. C. Yenen, I. Alanbay, N. K. Duru, andA. Has, imi, "CA19-9 may have clinica significance in mature cystic teratomas of the ovary," International Journal of Gynecological Cancer, vol.16, no. 1, pp. 189-193, 2006.

12. Bischof $P$ : What do we know about the origin of CA 125? Eur J Obstet Gynecol Reprod Biol, 1993; 49: 93-98

13. Jacobs IJ, Skates S, Davies AP et al: Risk of diagnosis of ovarian cancer after raised serum CA 125 concentration: a prospective cohort study. BMJ, 1996; 313: $1355-58$

14. Jacobs IJ, Skates SJ, Macdonald N et al: Screening for ovarian cancer: a pilot randomised controlled trial. Lancet, 1999; 353: $1207-10$

15. Kyung MS, Choi JS, Hong SH, and Kim HS, "ElevatedCA 19-9 levels in mature cystic teratoma of the ovary," International Journal of Biological Markers, vol. 24, no. 1, pp. 52-56, 2009. 